\title{
Conic Surveillance Evasion ${ }^{1}$
}

\author{
J. LEWIN ${ }^{2}$ AND G. J. OLSDER ${ }^{3}$ \\ Communicated by P. L. Yu
}

\begin{abstract}
A surveillance-evasion differential game of degree with a detection zone in the shape of a two-dimensional cone is posed. The nature of the optimal strategies and the singular phenomena of the value function are described and correlated to subsets of the space of all possible parameter combinations, showing the relation of the singular phenomena in differential game theory and control theory.
\end{abstract}

Key Words. Differential games, optimal strategies, optimal trajectories, singular surfaces, solution concepts, surveillance evasion.

\section{Introduction}

Differential Games of surveillance-evasion are used to model a conflict situation where a pursuer counteracts an evader in order to maintain the state of a dynamic system within a specified detection set in the state space.

In Ref. 1, quantitative solutions of a surveillance-evasion game of degree with a circular detection set have been described. The current paper discusses a problem with a detection set in the form of a two-dimensional semi-infinite cone symmetric about the velocity vector of the pursuer.

Like other two-dimensional nontrivial quantitative differential games, such as the homicidal chauffeur (Ref. 2), the solution exhibits various singular trajectories and singular manifolds across which the value function or its gradient have jump discontinuities.

The candidate solutions were constructed by methods developed by Isaacs (Ref. 3) and Breakwell, and it turns out that they satisfy the sufficient

\footnotetext{
${ }^{1}$ The first author was partially supported by the Stichting Hogeschoolfonds Twente, Enschede, The Netherlands.

${ }^{2}$ Senior Lecturer, Department of Aeronautics, Technion, Haifa, Israel.

${ }^{3}$ Senior Lecturer, Department of Applied Mathematics, Twente University of Technology, Enschede, The Netherlands.
} 
conditions for optimality (Ref. 4). The solutions relate to a modified solution concept (Refs. 5-6), within which optimal strategies never violate the information constraint that precludes the possibility that one player may know the current control choice of his opponent.

The first part describes the set up of the game model and gives some preliminary results. It is followed by a detailed description of the nature of the singular phenomena as the parameters of the problem take different values in the parameter space.

For certain parameter combinations and for a certain part of the state space, the evader can be kept under surveillance indefinitely. This part of the state space is surrounded by semipermeable surfaces, which the evader cannot cross without the pursuer's help. The same technique of enclosing an area by semipermeable surfaces, from which the evader cannot excape, has been used in Ref. 7.

\section{Statement of the Game Model}

An evader, called player $E$, with a velocity of magnitude 1 (normalized) and an infinite rate of turn tries to escape from inside a detection set, described as a two-dimensional semi-infinite cone with half angle $\bar{\theta}$ in a planar reference frame (relative frame) attached to the velocity vector of a pursuer. The pursuer, player $P$, with a velocity of magnitude $w$ and a minimum turn radius of length 1 (normalized) tries to maintain the evader within the detection set for maximum time. This game may be considered as a simplified model for planar surveillance where the evader is more maneuverable.

The system equations in Cartesian and polar forms are:

$$
\begin{aligned}
& \dot{x}=\sin \psi-y w \phi, \\
& \dot{y}=\cos \psi-w+x w \phi, \\
& \dot{r}=\cos (\psi-\theta)-w \cos \theta, \\
& r \dot{\theta}=\sin (\psi-\theta)+w \sin \theta-r w \phi .
\end{aligned}
$$

Player $E$ chooses $\psi$ at each instant in order to minimize the time at which the state of the system crosses the right or left target sets $\theta= \pm \bar{\theta}$ to have $|\theta| \leq \bar{\theta}$ for as short as possible. Player $P$ chooses $\phi$, subject to $|\phi| \leq 1$; for example, $\phi=+1$ is a full turn to the right. 


\section{Preliminary Results}

The part of the target sets at which player $E$ can guarantee that trajectories can cross the boundary and escape the detection set was termed by Isaacs usable part (UP).

We can easily find that, at $(r, \bar{\theta})$ satisfying

$$
r<\sin \bar{\theta}+1 / w
$$

the following holds:

$$
0<\max _{\psi} \min _{\phi} \dot{\theta}(r, \vec{\theta}) .
$$

Thus, (5) defines the UP of the right target set (segments PB in Fig. 1).

In regions where the gradient of the value function $T$ is continuous, Isaacs' main equation can be applied as a necessary condition either in its Cartesian or its polar representations:

$$
\begin{aligned}
& \min _{\psi} \max _{\phi}\left\{T_{x}(\sin \psi-y w \phi)+T_{y}(\cos \psi-w+x w \phi)+1\right\}=0 \\
& \min _{\psi} \max _{\phi}\left\{T_{r}[\cos (\psi-\theta)-w \cos \theta]\right. \\
& \left.\quad+\left(T_{\theta} / r\right)[\sin (\psi-\theta)+w \sin \theta-r w \phi]+1\right\}=0
\end{aligned}
$$

so that clearly, in the above-mentioned regions, the optimal strategies $\phi^{*}, \psi^{*}$ will satisfy: ${ }^{4}$

$$
\begin{gathered}
\phi^{*}=\operatorname{sign}\left(x T_{y}-y T_{x}\right)=-\operatorname{sign} T_{\theta}, \\
\left(\sin \psi^{*}, \cos \psi^{*}\right) \|\left(-T_{x},-T_{y}\right),
\end{gathered}
$$

"The symbol || in Eqs. (10)-(11) stands for "parallel to".

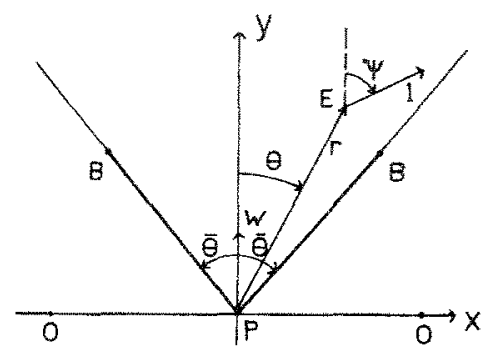

Fig. 1. Geometric definitions. 
or

$$
\left(\cos \left(\psi^{*}-\theta\right), \sin \left(\psi^{*}-\theta\right)\right) \|\left(-T_{r},-T_{\theta} / r\right) .
$$

In regions where $T$ is $C^{2}$, adjoint equations hold along the optimal trajectories:

$$
\begin{aligned}
& \dot{T}_{x}=-T_{y} w \phi^{*} \\
& \dot{T}_{y}=T_{x} w \phi^{*}
\end{aligned}
$$

or

$$
\begin{aligned}
& \dot{T}_{\theta}=-w\left[T_{r} \sin \theta+\left(T_{\theta} / r\right) \cos \theta\right]=-w T_{x}, \\
& \dot{T}_{r}=\left(T_{\theta} / r^{2}\right)[\sin (\psi-\theta)+w \sin \theta] .
\end{aligned}
$$

For terminal states $\left(r_{f}, \bar{\theta}\right)$ that belong to the usable part (UP), we have

$$
\begin{gathered}
T_{r}\left(r_{f}, \bar{\theta}\right)=0, \\
T_{\theta}\left(r_{f}, \bar{\theta}\right)=-r_{f} /\left(1+w \sin \bar{\theta}-r_{f} w\right) .
\end{gathered}
$$

We can now use $(16),(17)$ to determine $\phi^{*}, \psi^{*}$ in the vicinity of the UP, and we are able to integrate the system and the adjoint equations and obtain candidate optimal paths that emanate backward in retrograde time $\tau$.

We find for example that, for a path emanating from $\left(r_{f}, \bar{\theta}\right)$,

$$
\begin{gathered}
\sin \psi^{*}(\tau)=\cos (\bar{\theta}+w \tau), \\
\cos \psi^{*}(\tau)=-\sin (\bar{\theta}+w \tau), \\
\phi^{*}=+1, \\
x=1-\cos (w \tau)+r_{f} \sin (\bar{\theta}+w \tau)-\tau \cos (\bar{\theta}+w \tau), \\
y=\sin (w \tau)+r_{f} \cos (\bar{\theta}+w \tau)+\tau \sin (\bar{\theta}+w \tau), \\
-T_{\theta}=\frac{r_{f}+\sin (\bar{\theta}+w \tau)-\sin \bar{\theta}}{1+w \sin \bar{\theta}-r_{f} w}=\frac{r \cos (\bar{\theta}-\theta+w \tau)}{1+w \sin \bar{\theta}-r_{f} w}
\end{gathered}
$$

The isochrones (surfaces of equal time to go) happen to be straight lines that are inclined at an angle $\bar{\theta}+w \tau$ to the $y$-axis and are tangent to a circle of radius $\tau+\cos \bar{\theta}$, centered at 0 , which is the turn center of player $P$ (see Fig. 1).

The equations for the $\tau$-isochrone are

$$
x \cos (\bar{\theta}+w \tau)-y \sin (\bar{\theta}+w \tau)+\tau+\cos \bar{\theta}-\cos (\bar{\theta}+w \tau)=0,
$$

or

$$
-r \sin (\bar{\theta}-\theta+w \tau)+\cos \bar{\theta}-\cos (\bar{\theta}+w \tau)+\tau=0
$$

Equations (18)-(25) are not valid after (23) vanishes, i.e., for

$$
\tau>(\pi / 2+\theta-\bar{\theta}) / w .
$$




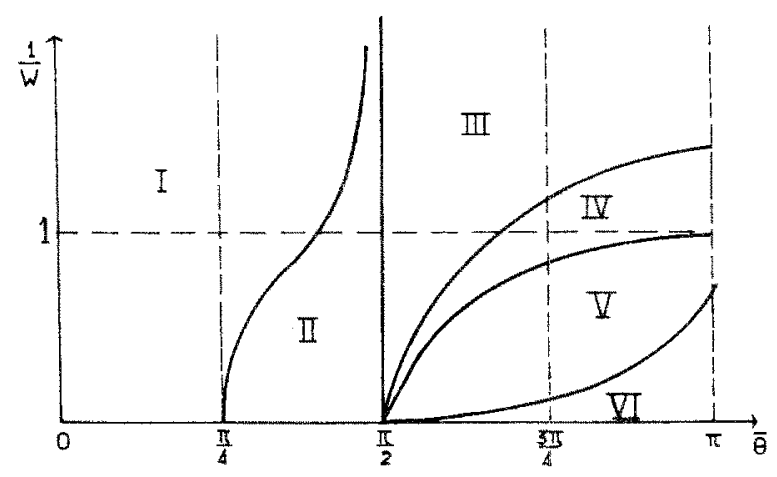

Fig. 2. Partition of the parameter space.

The optimal paths (21), (22) have a clear geometric representation in the real space. Near termination, player $P$ moves on a circular arc that corresponds to a full right turn $\phi^{*}=+1$, while player $E$ moves along a straight line that is perpendicular to the target boundary at the instance of $E$ 's escape. Moreover, $T_{\theta}$ vanishes on such a path (indicating a possible switch of $\phi^{*}$ ), when the velocity vector of player $E$ in the real space points directly at $P$.

As is often the case in the course of the solution of nontrivial differential games, the paths constructed backwards from the usable part fail to fill the game space $-\bar{\theta} \leq \theta \leq \bar{\theta}$ with candidate optimal paths. Moreover, paths eventually intersect. This is a clear indication that a solution of our game, if it exists, may exhibit discontinuities in the value function $T$ and its gradient across various manifolds in the game space.

\section{Parameter Space}

The nature of the solutions was found to be dependent on two parameters: $w$ and $\bar{\theta}$ (speed ratio and cone angle). In the remaining parts of this work, the space of all possible parameter paths $(w, \bar{\theta})$ will be partitioned into subsets, and the nature of the singular phenomena of the solutions will be presented for typical parameter values $(w, \bar{\theta})$ for each such subset. The cases for which $w \ll 1$ and $w \gg 1$ also belong to the parameter space, but they really constitute degenerate games in which one player is nonmoving. The solutions of the optimal control problems that correspond to these degenerate cases will be described, and they will serve to illuminate the relationship between singular phenomena in differential games and in optimal control theory.

Table 1 summarizes the singular phenomena encountered in the various subsets of the parameter space described in Fig. 2. 
Table 1. Summary of singular phenomena.

\begin{tabular}{|c|c|c|c|c|c|c|c|}
\hline Type of singular surface & Designation & I & II & III & IV & $\mathrm{V}$ & VI \\
\hline Evader's dispersal line & EDL & + & + & & & & \\
\hline Pursuer's dispersal line & PDL & + & + & + & + & + & + \\
\hline Switch surface & TS & & + & + & + & + & + \\
\hline Equivocal surface & EV & + & + & & & & \\
\hline Pursuer's state constraint & PSC & + & + & + & + & & \\
\hline Barrier & B & & & + & + & + & + \\
\hline Pursuer's barrier state constraint & PBSC & & & & + & + & \\
\hline Closed barrier & $\mathrm{CB}$ & & & & & + & + \\
\hline
\end{tabular}

\section{Solutions in Region I and II: $\bar{\theta} \leq \pi / 2$}

Case (Ia). $\quad w \gg 1, \bar{\theta} \leq \pi / 4$, Nonmoving Evader. The case $w \rightarrow \infty$ corresponds to a nonmoving evader. The game degenerates to a problem of optimal control. Figure 3 describes the optimal strategies and the corresponding optimal trajectories for this case.

The switch curves TS (transition surfaces) are circular arcs about the centers of player $P$ 's right turns and left turns. We observe that the TS is also an optimal trajectory along which the state moves after the switch. The $y$-axis is a dispersal line (PDL), across which the gradient of the value

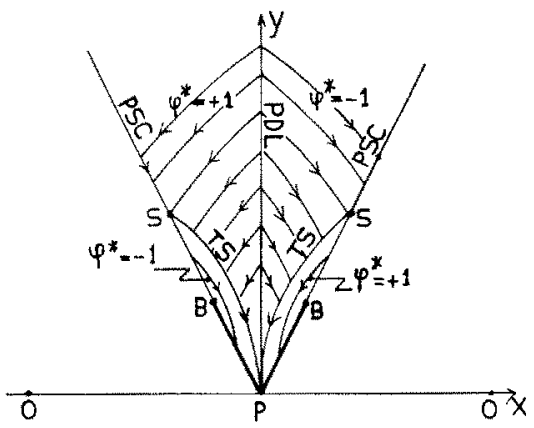

Fig. 3. Case (Ia), $w \gg 1, \bar{\theta} \leq \pi / 4$. 


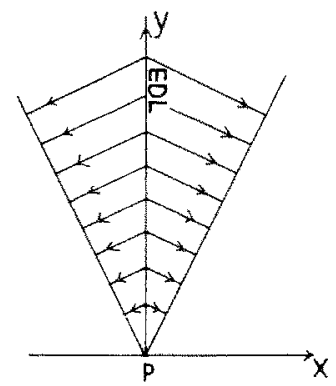

Fig. 4. Case (lb), $0 \leq w<1, \theta \leq \pi / 2$.

function is discontinuous. On the $y$-axis, player $P$ can choose either direction of turn, so that optimal trajectories leave the PDL to both its sides.

The portion $r \geq 2 \sin \bar{\theta}=r_{s}$ of the target $\operatorname{set} \theta=\bar{\theta}$ constitutes a singular arc that hugs the boundary as a state constraint (PSC). Along this arc, $P$ uses an intermediate value of $\phi$ determined by

$$
\tilde{\phi}=\sin \bar{\theta} / r \text {. }
$$

Note that the paths that reach the PSC are not tangent to it, and hence the switch function for $\phi$ necessarily vanishes at their junction with the PSC arc.

Case (Ib). $0 \leq w \ll 1, \bar{\theta} \leq \pi / 2$, Nonmoving Pursuer. The case $w \rightarrow 0$ corresponds to a nonmoving pursuer. The trivial solution of the resulting control problem is described in Fig. 4.

Case (Ic). Figure 5 describes optimal strategies, optimal trajectories, and singular surfaces of a typical nondegenerate game for parameters $(w, \bar{\theta})$ in region $\mathrm{I}$.

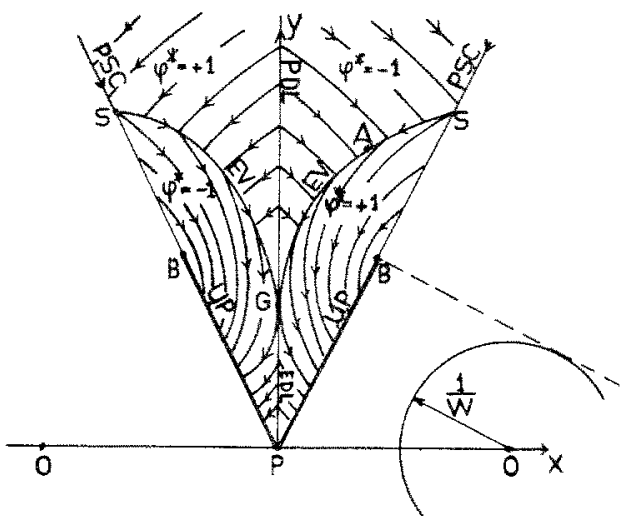

Fig. 5. Case (Ic). 
The point $\mathrm{G}$ (graze point) is the unique point where a path that ends in the UP is tangent to the $y$-axis. From the point $G$, a singular corner surface starts (Ref. 4), which Isaacs named (Ref. 3) equivocal surface (EV), across which the gradient of the value function is discontinuous.

The EV has been elucidated in Fig. 6, in which the vectogram at point $A$ of Fig. 5 has been drawn. Before the trajectory reaches the EV (forward time direction), the velocity vector is $\overrightarrow{\mathrm{AM}}$. The trajectory reaches the $\mathrm{EV}$ at point $A$, at which there are two possibilities: the velocity vector becomes either $\overrightarrow{\mathrm{AN}}$ or $\overrightarrow{\mathrm{AR}}$. The projections along the normal to the isochrone at point $\mathrm{A}$ [Eq. (25)] are the same, and hence both possibilities are equally good. If the evader $E$ chooses $\psi$ along SR, the pursuer $P$ will choose $\phi^{*}=+1$, and the resulting trajectory leaves the $\mathrm{EV}$ in the direction of $\overrightarrow{\mathrm{AR}}$. If $E$ chooses $\psi$ along SQ (i.e., $\psi=\theta+\pi$ ), then $P$ will choose $\phi$ such that the direction of the velocity vector is along the $E V$.

We can obtain the right EV-curve by integrating the following differential equation starting from $\mathrm{G}$ :

$$
(1 / r)(d r / d \theta)=\frac{-(1+w \cos \theta) \cos (\bar{\theta}-\theta+w \tau)}{(w \sin \theta-r w) \cos (\bar{\theta}-\theta+w \tau)+1-\sin (\theta-\theta+w \tau)},
$$

where $\tau$ is found, for each $(r, \theta)$, through (25). Along EV, player $P$ uses $\tilde{\phi}$ from

$$
1-\tilde{\phi}=\frac{1-\sin (\bar{\theta}-\theta+w \tau)}{r w \cos (\bar{\theta}-\theta+w \tau)}
$$

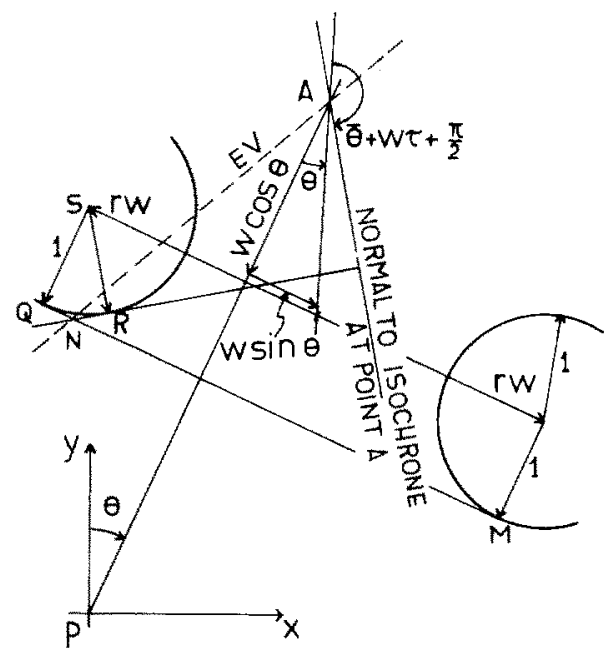

Fig. 6. Vectogram at equivocal surface. 
These equations were derived by requiring that, if player $E$ chooses either to move optimally along the EV or to move in the $\phi^{*}=+1$ field, he should be traversing the isochrones of the $\phi^{*}=+1$ field at the same time rate.

From (29), we see that $\tilde{\phi}=+1$ when

$$
\bar{\theta}-\theta+w \tau=\pi / 2 \text {. }
$$

The EV cannot be constructed any further, since

$$
|\phi| \leq 1 \text {. }
$$

Region I in the parameter space is defined to be the set of $(w, \bar{\theta})$ for which the equivocal surface ends on the target set $\theta=\bar{\theta}$ at a point $S$ before (30) is attained.

From the theory of junction conditions to corner surfaces (Ref. 4), we conclude that the optimal strategy $\psi^{*}$ of player $E$ on the paths that reach the equivocal surface are continuous across the junction. Moreover, they arrive at the EV nontangentially, and hence the switch function for $\phi$ necessarily vanishes at the junction. In view of the preliminary results, this means that, at the junction,

$$
\psi^{*}=\theta+\pi
$$

Eq. (31) enables us to construct the field of extremals that reach the EV.

The portions $r>r_{s}$ of the target set $\dot{\theta}=\bar{\theta}$ again form a singular arc. Indeed, along this arc, player $P$ dominates $\dot{\theta}$ and can provide a control $\tilde{\phi}(\psi)$ for any $\psi$ so that the trajectory will follow $\theta=\bar{\theta}$. Player $E$ 's optimal policy is to maximize $-\dot{r}$ by using

$$
\tilde{\phi}=\theta+\pi \text {. }
$$

We name this singular arc pursuer's state constraint (PSC). The optimal strategy $\psi^{*}$ of player $E$ on the paths that reach the PSC is continuous across the junction (Ref. 4), enabling us to construct the field of extremals that arrive at the PSC. By (32), the switch function for $\phi$ vanishes at the junction, so that at $S$ the same path arrives at the PSC and at the EV providing a smooth piecing of the fields of extremals that reach the EV and the PSC.

The $y$-axis is a dispersal surface across which the gradient of the value function is discontinuous. Notice that, above $\mathrm{G}$, the pursuer chooses which side the trajectory goes, and we name it pursuer's dispersal line (PDL). Below G, it is evader's choice, and it is called evader's dispersal line (EDL).

The game space is thus filled with candidate optimal trajectories, and the solution satisfies the sufficient conditions for optimality.

In the course of the derivation of the paths backward from singular surfaces, we made use of the theory of junction conditions. The reader is referred to Refs. 1-3 for more examples of their use and to Ref. 4 for a summary of the theory. 


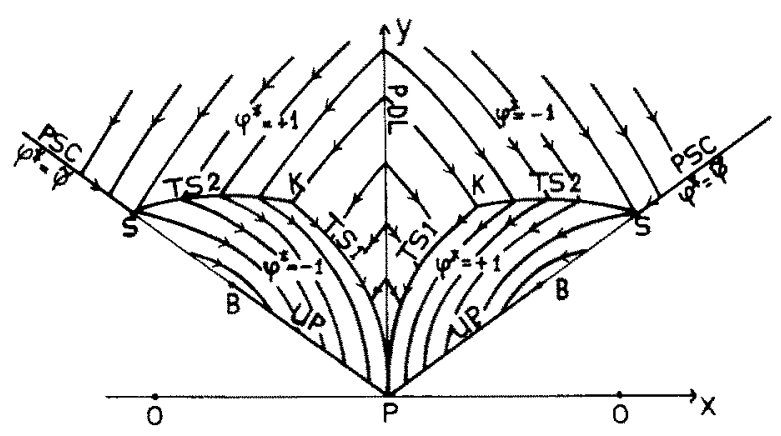

Fig. 7. $w \gg 1, \pi / 4 \leq \bar{\theta} \leq \pi / 2$.

It is also important to remember that the singular surface $\mathrm{EV}$ was constructed by its characteristic differential equation, rather than by observing discontinuities in $\nabla T$ numerically. We should notice that the use of a strategy of the type $\tilde{\phi}(\psi)$ to maintain a singular arc is nonadmissible, since we assume that the players are not notified of their opponents' current controls choices. However, if a player can guarantee any desired proximity to a singular arc and to the value on it, then within an extended solution concept, we accommodate the singular arc as a limit arc for $\epsilon$-optimal paths generated by admissible strategies. For further discussion, the reader is referred to Ref. 5 and Ref. 6.

Case (IIa). $\quad w \gg 1, \pi / 4 \leq \bar{\theta} \leq \pi / 2$, Nonmoving Evader. Like Case (Ia), this is a degenerate game with a nonmoving evader. Figure 7 gives optimal strategies and optimal trajectories for this case.

The switch curve is divided into two parts. TS1 is a switch curve as well as an optimal path similar to TS in Case (Ia) (see Fig. 3). TS2 is an ordinary switch curve, which does not constitute a trajectory. TS1 and TS2 join at points $\mathrm{K}$ for which

$$
\begin{gathered}
r_{\mathrm{K}}=2 \cos \bar{\theta} \\
\theta_{\mathrm{K}}= \pm(\pi / 2-\bar{\theta})
\end{gathered}
$$

The $y$-axis is again a pursuer's dispersal line (PDL), and pursuer state constraint (PSC) singular arcs exist along the lines $\theta= \pm \bar{\theta}$ respectively beyond the points $\mathrm{S}$ like in Case (Ia).

Case (IIb). $\pi / 4 \leq \bar{\theta} \leq \pi / 2$. Figure 8 presents optimal strategies, optimal trajectories, and singular surfaces for a solution of a typical nondegenerate game with parameters $(w, \bar{\theta})$ in Region II of the parameter space 


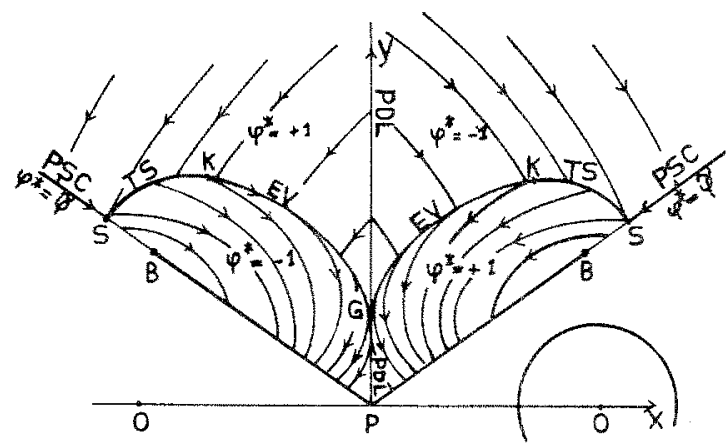

Fig. $8 . \quad \pi / 2 \geq \bar{\theta} \geq \pi / 4$.

(Fig. 2). We observe that, in this case, switch curves TS appear that end on points $\mathrm{S}$ on the target set. The TS is given by

$$
r=\cos \bar{\theta}+\sin \theta+(\theta-\bar{\theta}+\pi / 2) / w \text {. }
$$

At the instant of switch, we have by (26)

$$
\theta=w \tau+\bar{\theta}-\pi / 2
$$

Comparing (36) and (30), we see that the equivocal surfaces EV end at points $K$ that belong to the switch surfaces TS.

Moreover, by (31), the switch function of $\phi$ vanishes on the path that reaches $K$ at the junction, so that the fields of paths that reach the $E V$ and the TS piece smoothly.

As in Case (Ic), pursuer state consiraint (PSC) singular arcs exist along the lines $\theta= \pm \bar{\theta}$ beyond the points $\mathrm{S}$. By (32), the switch function of $\phi$ vanishes on the path that reaches $S$ at the junction, so that the field of paths that reach the PSC and the TS piece together.

The point $\mathrm{G}$ again divides the $y$-axis into a pursuer dispersal line (PDL) and an evader dispersal line (EDL).

\section{Solutions in Regions III-VI: $\bar{\theta} \geq \pi / 2$}

Barriers. For $\bar{\theta}>\pi / 2$ and any $w$, the solutions exhibit the phenomenon of barriers. Manifolds across which the value function is discontinuous terminate on $\theta= \pm \bar{\theta}$ at the point $B$ which is the boundary of the usable part (UP).

The barriers can be constructed from B by either using the preliminary results about the paths emanating backward from the usable part or by a 


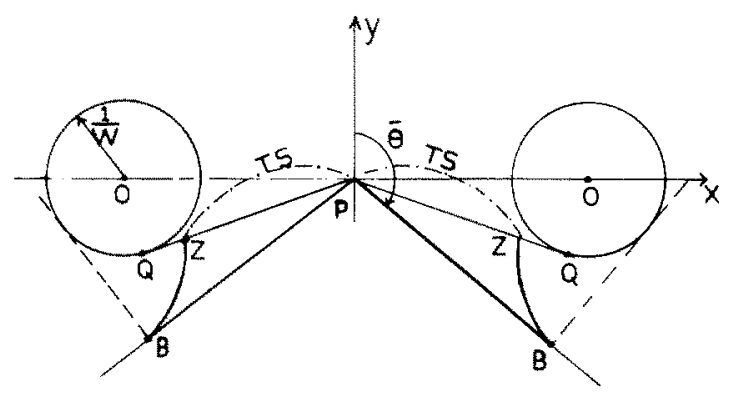

Fig. 9. $w \sin (\bar{\theta}-\pi / 2) \geq \pi-\bar{\theta}+\sin ^{-1}(1 / w)$, Region VI.

game of kind approach utilizing the property that a barrier is necessarily a semipermeable surface (Ref. 3).

We find that the motion in the real space that corresponds to the barrier in the vicinity of $B$ is a straight motion of player $E$ along a line tangent to a circle with a radius of magnitude $1 / w$ centered at 0 along with a full turn by player $P$.

It is easy to see, in a manner analogous to Refs. 1-3, that the barrier can be described geometrically by an evolute that starts at $\mathrm{B}$ and winds the $1 / w$-circle. Beyond the point $C$ (Fig. 11), where the evolute reaches the $1 / w$-circle, the construction fails! Because the angular velocities match at $\mathrm{C}$, both $\dot{r}=0$ and $\dot{\theta}=0$ on the barrier, indicating a cusp or, equivalently, a nonsmooth behavior of the normals to the surface. Beyond such a cusp, our construction method, which is based on the assumption of smooth normals, is nonvalid.

It was found that whether the barrier intersects a tangent to the $1 / w$-circle or not dominates the nature of the solution. The following regions in the parameter space $(w, \bar{\theta})$ can be defined with respect to this property.

In the region VI (see Fig. 2) defined by

$$
w \sin (\bar{\theta}-\pi / 2) \geq \pi-\bar{\theta}+\sin ^{-1}(1 / w),
$$

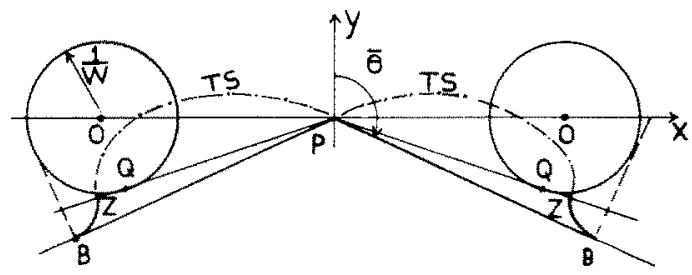

Fig. 10. $1 \leq w \sin (\bar{\theta}-\pi / 2) \leq \pi-\bar{\theta}+\sin ^{-1}(1 / w)$, Region $V$. 


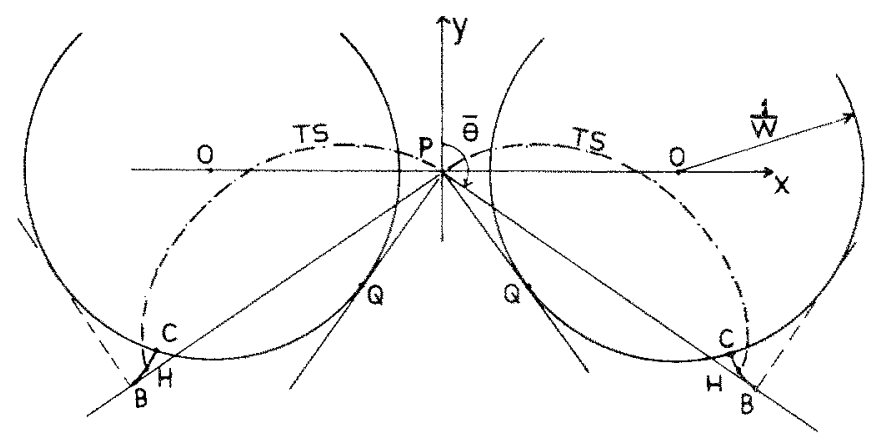

Fig. 11. $(\pi / 2-1) \leq w \sin (\bar{\theta}-\pi / 2) \leq 1$, Region IV.

the barrier intersects the tangent $P Q$ (Fig. 9) between $P$ and $Q$.

In the region $\mathrm{V}$ (see Fig. 2), defined by

$$
1 \leq w \sin (\bar{\theta}-\pi / 2) \leq \pi-\bar{\theta}+\sin ^{-1}(1 / w),
$$

the barrier intersects the tangent PQ (Fig. 10) beyond $\mathrm{Q}$.

In the regions III and IV (see Fig. 2) defined by

$$
w \sin (\bar{\theta}-\pi / 2) \leq 1 \text {, }
$$

the barrier does not intersect PQ (Figs. 11 and 12).

Switch Curve. A switch curve (TS) exists in the solution when $\bar{\theta} \geq \pi / 2$ for all values of $w$. The TS is given by Eq. (35). For parameter values $(w, \bar{\theta})$ in regions $I V, V, V I$, the switch curves reach the barrier at the points $\mathrm{Z}$ or $\mathrm{H}$ (see Figs. 9, 10, 11). For parameters in region III, which is defined by

$$
w \sin (\bar{\theta}-\pi / 2) \leq \pi / 2-1,
$$

the switch curves do not intersect the barriers, but reach the target set at the points S (see Fig. 12).

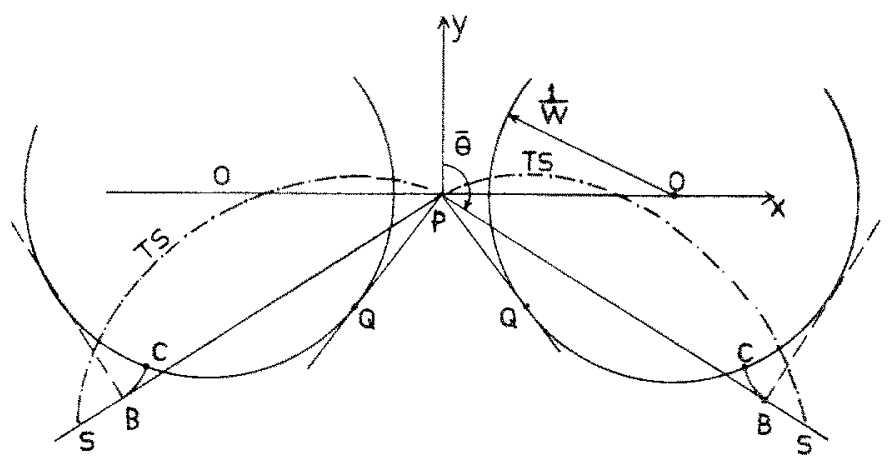

Fig. 12. $w \sin (\bar{\theta}-\pi / 2) \leq \pi / 2-1$, Region III. 


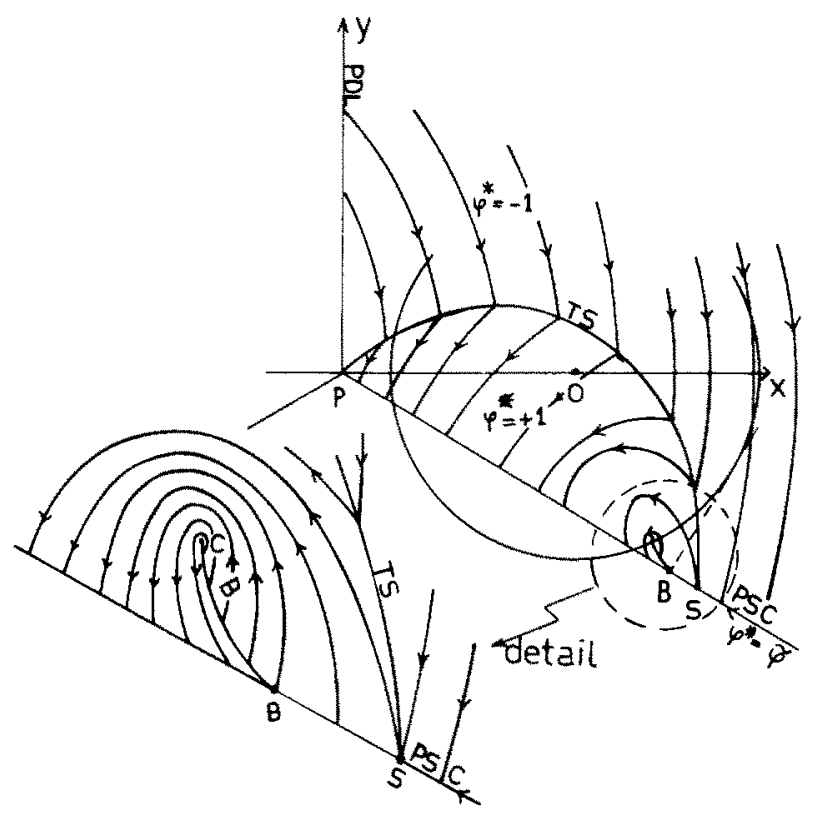

Fig. 13. $w \sin (\bar{\theta}-\pi / 2) \leq \pi / 2-1$, Case III.

Case (III). $\pi / 2-1 \geq w \sin (\bar{\theta}-\pi / 2)$. Some features of the solution of the game for parameter values $(w, \bar{\theta})$ in region III are shown in Fig. 13.

A switch curve starts at $P$ and ends at the point $S$ on the target set $\theta=\bar{\theta}$, beyond which a PSC singular arc exists. The $y$-axis is again a dispersal surface. A barrier emanates from the boundary of the usable part $B$ and ends at the cusp point $C$ (see also Fig. 12). The value function has a jump discontinuity (between finite values) across the barrier.

It is clear that, in Case (III), player $E$ can escape surveillance from all starting points.

As $w$ decreases, points $B$ and S get farther away along the line $\theta=\bar{\theta}$. For $w \ll 1$, the solution becomes trivial as shown in Fig. 14 .

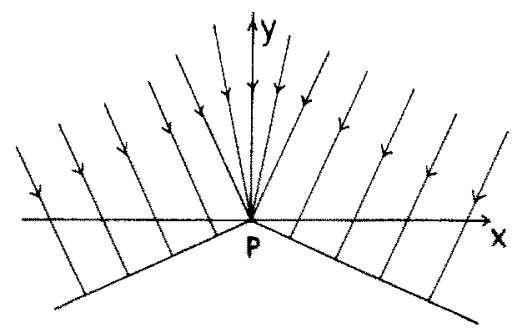

Fig. $14, w \ll 1$, nonmoving pursuer, $\bar{\theta}>\pi / 2$. 


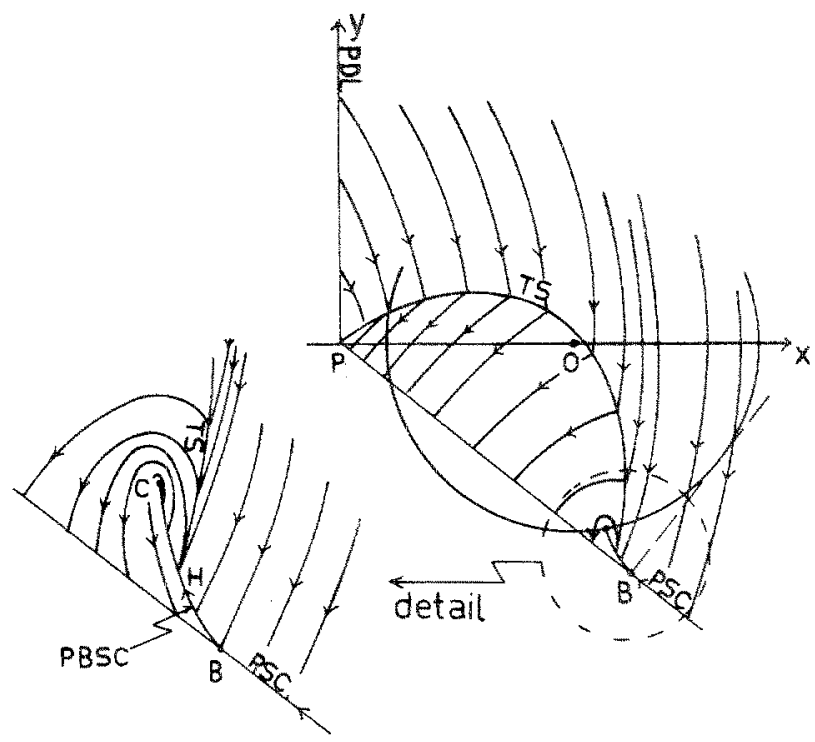

Fig. 15. $1 \geq w \sin (\bar{\theta}-\pi / 2) \geq \pi / 2-1$, Case IV.

Case (IV). $1 \geq w \sin (\bar{\theta}-\pi / 2) \geq \pi / 2-1$. Figure 15 represents a typical solution in region IV of the parameter space. Here, the switch curve TS meets the barrier at a point $\mathrm{H}$ giving rise to another type of singular arc. The barrier is necessarily a semipermeable surface. The TS always hits the barrier for Case (IV), which can be shown as follows. The cusp coordinates $\theta_{c}, t_{c}$ satisfy [see (3), (4)]

$\cos \psi \cos \theta_{c}+\sin \psi \sin \theta_{c}-w \cos \theta_{c}=\sin \left(\theta_{c}-\bar{\theta}-w \cos \bar{\theta}\right)-w \cos \theta_{c}=0$, $\sin \psi \cos \theta_{c}-\cos \psi \sin \theta_{c}+w\left(\sin \theta_{c}-r_{c}\right)$

$$
=\cos \left(\theta_{c}-\bar{\theta}-w \cos \bar{\theta}\right)+w\left(\sin \theta_{c}-r_{c}\right)=0 .
$$

For $\theta=\theta_{c}$, the $r$ for the switch becomes [see (35)]

$$
r_{s}=\cos \bar{\theta}+\sin \theta_{c}+\left(\theta_{c}-\bar{\theta}+\pi / 2\right) / w .
$$

It is straightforward to show that $r_{c}<r_{s}$, and hence TS hits the barrier. Player $P$ can thus prevent player $E$ from crossing the barrier from above it. Moreover, for any control $\psi$ used by player $E$, player $P$ can choose a control $\tilde{\phi}(\psi)$ so as to carry the path along the barrier. On this arc, player $E$ maximizes $(-\dot{r})$ by choosing

$$
\tilde{\psi}=\theta+\pi
$$

so the path traverses the barrier upward. At the point $H$, where the TS meets the barrier, player $P$ will play $\phi=+1$, and the path will not follow the barrier 


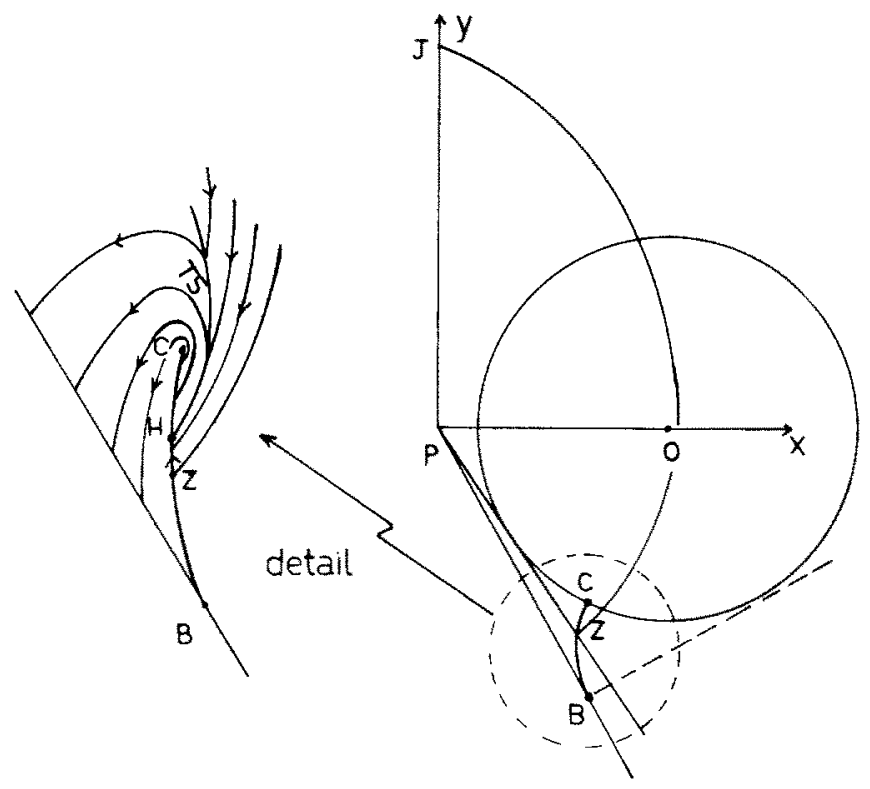

Fig. 16. $1 \leq w \sin (\bar{\theta}-\pi / 2) \leq \pi-\bar{\theta}+\sin ^{-1}(1 / w)$.

any further. The arc BH of the barrier constitutes a singular arc which is termed PBSC (pursuer barrier state constraint).

It is to be noticed that, as an optimal path follows the barrier upward toward $\mathrm{H}$, players $P$ and $E$ use strategies that are different from the barrier strategies by which the barrier was constructed.

The strategy $\psi$ of player $E$ is continuous along the path that reaches the PBSC at the junction. By (41), the switch function for $\phi$ vanishes at the junction, so that the field of extremals emanating backward from the PBSC fits smoothly with the fields of trajectories emanating from the TS and the PSC as argued before. The reader should note that the discussion made for Case (Ic) about the admissibility of strategies of the type $\tilde{\phi}(\psi)$ applies here also. As shown in Fig. 15, the solution contains PDL that are basically similar to those encountered in Case (III), so clearly player $E$ can escape from all starting points here, too.

Case V. $1 \leq w \sin (\bar{\theta}-\pi / 2) \leq \pi-\bar{\theta} \sin ^{-1}(1 / w)$. Some details of the solution in region $\mathrm{V}$ are depicted in Fig. 16. Along the arc BZ, not only can player $P$ push the path to the barrier, but he can quarantee $\dot{r} \geq 0$ as well.

Through the point $Z$, another semipermeable surface was constructed. It corresponds to a full left turn by player $P$ and a motion along a line in the real space that is tangent to the left $1 / w$-circle by player $E$. 
In order for the union of $\mathrm{ZJ}$ and $\mathrm{BZ}$ to qualify as a composite semipermeable surface, it has to satisfy certain corner conditions (Ref. 8) that guarantee that the corner $\mathrm{Z}$ does not leak. Indeed, at $\mathrm{Z}$,

$$
\tilde{\psi}=\theta+\pi,
$$

and the switch function for $\phi$ vanishes. This suffices to satisfy the corner conditions (Ref. 8); and JZB forms a composite closed barrier.

From starting points inside the closed barrier, player $E$ can escape in finite time. Outside the closed barrier, player $P$ can maintain surveillance indefinitely.

The optimal strategies and optimal trajectories inside the closed barrier closely resemble Case (IV) and will not be described in detail here.

Outside the closed barrier, it suffices for player $P$ to play optimally only if a path arrives at a barrier and to play in such a way as to maintain player $E$ under surveillance indefinitely. The reader is referred to Ref. 1 for another case of a composite barrier.

Case (VIa). $w \sin (\bar{\theta}-\pi / 2) \geq \pi-\bar{\theta}+\sin ^{-1}(1 / w)$. For parameters $(w, \bar{\theta})$ in region VI in the parameter space, the solution (see Fig. 17) exhibits a smooth composite barrier JZB. Inside the closed barrier, player $E$ can guarantee escape, while outside the barriers player $P$ can maintain player $E$ under surveillance indefinitely. At $Z$, a proper switch occurs on the barrier path, so that $Z$ also belong to the TS.

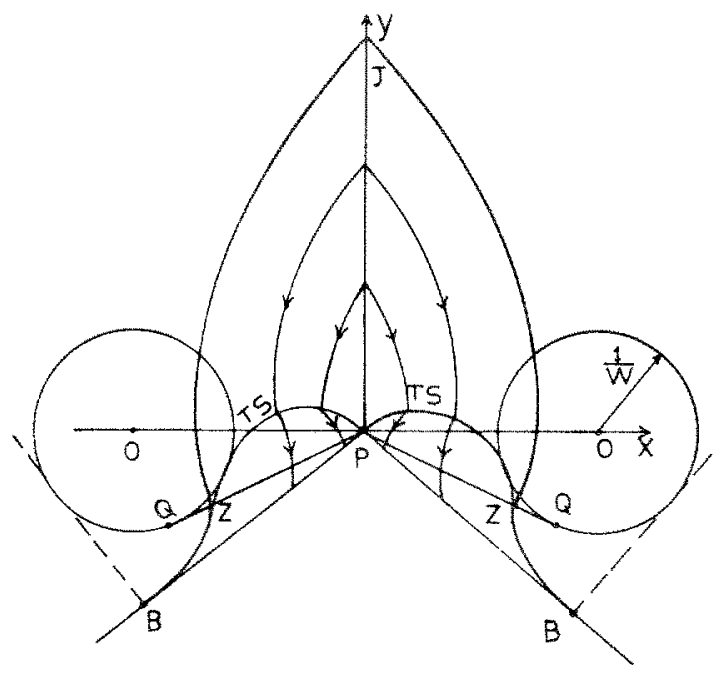

Fig. 17. $w \sin (\bar{\theta}-\pi / 2) \geq \pi-\bar{\theta}+\sin ^{-1}(1 / w)$. 


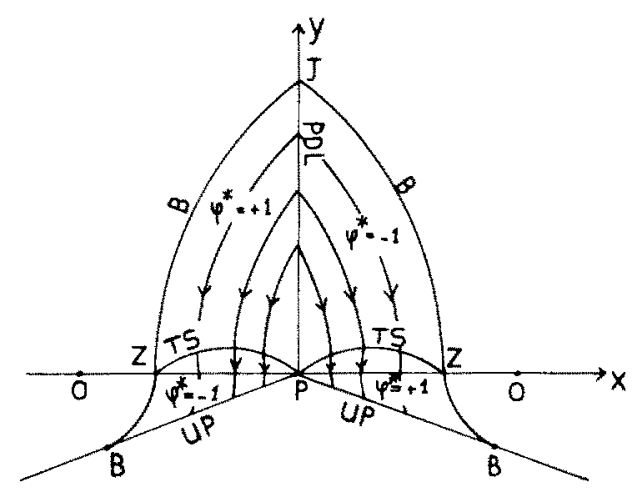

Fig. 18. $w \gg 1$, nonmoving evader, $\bar{\theta}>\pi / 2$.

Case (VIb). $w \gg 1$. The features of the relatively simple pattern of the solution of Case (VIa) (Fig. 17) remain almost the same even in the extreme case of a nonmoving evader $w \gg 1$ (see Fig. 18).

\section{Concluding Remarks}

The cone surveillance-evasion game of degree adds to the as yet too small number of solved nontrivial differential games of degree. We were somewhat lucky to have been able to show that the characterization of the singular surfaces can indeed be made almost independently of numerical procedures. Though an existence theory for nontrivial games of degree is not yet available, this problem shows that solutions are sometimes feasible. Further research could be aimed at solving nontrivial games of higher dimensions.

\section{References}

1. Lewin, J., and BREAKwell, J. V., The Surveillance-Evasion Game of Degree, Journal of Optimization Theory and Applications, Vol. 16, Nos. 3/4, 1975.

2. MERZ, A. W., Homicidal Chauffeur, A Differential Game, Stanford University, Guidance and Control Laboratory, Report No. SUDAAR 418, 1971.

3. ISAACS, R., Differential Games, 2nd Edition, John Wiley and Sons, New York, New York, 1976.

4. BERNHARD, P., Singular Surfaces in Differential Games, Differential Games and Applications, Edited by P. Hagedorn, H. W. Knobloch, and G. J. Olsder, Springer-Verlag, Berlin, Germany, 1977. 
5. LEWIN, J., The Bang-Bang-Bang Problem Revisited, Journal of Optimization Theory and Applications, Vol. 18, No. 3, 1976.

6. LEWIN, J., On Guaranteed Termination in Differential Games (to appear).

7. OLsDer, G. J, and BREAKwell, J. V., Role Determination in an Aerial Dogfight, International Journal of Game Theory, Vol. 3, No. 1, 1974.

8. BERNHARD, P., Conditions de Coin pour les Jeux Differentiels, Seminaire sur les Jeux Differentiels, Centre D'Automatique, Paris, France, 1971. 